\title{
Preparation of a New Sol-Gel Molecularly Imprinted Polymer with Isatin Template for an On-Line Solid- Phase Extraction of HMF from Fruit Juice Samples
}

\author{
Yalda Pasandideh* \\ Department of Chemistry, Karaj Branch, Islamic Azad University, Alborz, Iran \\ *Corresponding author: Yalda Pasandideh, Department of Chemistry, Karaj Branch, Islamic Azad University, Alborz, Iran. \\ To Cite This Article: Yalda Pasandideh. Preparation of a New Sol-Gel Molecularly Imprinted Polymer with Isatin Template for an On-Line Solid- \\ Phase Extraction of HMF from Fruit Juice Samples. Am J Biomed Sci \& Res. 2022 - 15(2). AJBSR.MS.ID.002093. \\ DOI: 10.34297/AJBSR.2022.15.002093
}

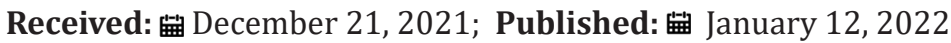

\begin{abstract}
5-hydroxymethylfurfural (HMF) is an aromatic compound that is an admitted indicator of reduced quality in different foodstuffs. Here, a novel, sensitive, and simple online solid-phase extraction (SPE) based on a molecularly imprinted sol-gel polymer (MIS) coupled to an HPLC-UV was designed and developed for the extraction and determination of HMF. Under optimum conditions, good linearity was achieved from 0.03 to $0.45 \mathrm{\mu g} \mathrm{ml}^{-1}$ with relative standard deviations (RSDs) below 5.17\%. The Limit of detection (LOD) was $0.023 \mu \mathrm{g} \mathrm{ml}^{-1}$, and the recoveries of spiked real samples were more than $97 \%$. The experimental results demonstrated that the proposed procedure is not to be affected by the matrix interfaces and can be successfully utilized for the routine analysis of HMF in different samples.
\end{abstract}

Keywords: Fruit Juice; HMF; Molecularly Imprinted Polymer; Sol-gel; Online Solid-Phase Extraction.

\section{Introduction}

In analytical chemistry, sample preparation refers to a group of methods in which a sample is prepared for specific chemical analysis or improved the conditions of that analysis [1]. This step is usually the most time-consuming part of an analytical analysis and can significantly affect the obtained data [2]. Liquidliquid extraction (LLE), solid-phase extraction (SPE), and solidphase microextraction (SPME) are some of the most popular sample preparation methods [3]. SPE, that also called liquid-solid extraction, is a technique used for clean-up, trace enrichment, extraction, isolation, and separation of different analytes from liquid matrixes. Generally, in this technique, an aqueous sample passes through in a solid phase and there after extracts by a suitable organic solvent [4]. Today, broad ranges of adsorbents (silica based and polymeric phases) are commercially available to improve the selectivity and simplify the SPE method [5]. However, there is still a need to synthesize and produce specific phases for a particular analyte/analytes extraction. In recent past years, using of molecularly imprinted polymers (MIPs) and molecularly imprinted sol-gel materials (MIS) have been significantly extended for the preparation of high-selective sorbents to improve the SPE techniques selectivity. Polymers synthesized by the sol-gel processes are prepared under gentle conditions, possess high chemical and temperature resistance, and are very suitable for online SPE [6]. 5-Hydroxymethylfurfural (HMF) is considered a potential cytotoxic, genotoxic, and carcinogenic for humans and is a sign of quality deterioration in a wide scope of foodstuffs [7]. Therefore, its trace determination in foodstuffs is really essential. The present paper introduces a new MIS polymer by combining the imprinting technique with a sol-gel process utilizing isatin as a dummy template. The synthesized sorbent was used to develop a sensitive and rapid online SPE technique for the selective extraction 
and determination of traces amount of HMF in apple and cherry juice samples.

\section{Experimental}

\section{Chemicals and Materials}

5-(Hydroxymethyl) frfural (HMF), tetrahydrofuran(THF) and tetraethylorthosilicate (TEOS) were bought from SigmaAldrich (St. Louis, USA). Isatin, all HPLC-grade solvents and the other chemicals and reagents were from Merck (Darmstadt, Germany). A stock solution of HMF was prepared in ethyl acetate and then the working solutions were prepared by an appropriate dilution with deionized water acidified with $\mathrm{HCl}$ at $\mathrm{pH}=4.0$. All the solutions were wrapped in an aluminum foil and stored below $4^{\circ} \mathrm{C}$. A mixture if H2O: THF $(80: 20 \mathrm{v} / \mathrm{v})$ at the flow rate of $1 \mathrm{~mL} \mathrm{~min}^{-1}$ was utilized as the mobile phase for the HMF elution of in isocratic mode. The commercial samples were purchased from a local store.

\section{Instrument and Apparatus}

A Perkin-Elmer chromatographic device (CA, USA) equipped with a binary pump (model 200), an UV-detector (model 200), and a Rheodyne six-port valve was employed for the high-performance liquid chromatographic (HPLC) analysis. The Total Chrom software processed the spectral and chromatographic information obtained from detector. The SPE mini-column was an $10 \mathrm{~mm}$ i.d. $\times 20 \mathrm{~mm}$ long stillness steel column with proper frits at both sides. All separations were performed on an analytical C18 column (Machery-Nagel, $5 \mu \mathrm{m}$, $4.6 \mathrm{~mm}$ i.d. $\times 25 \mathrm{~cm}$ long, $5 \mu \mathrm{m}$ pore size). All the analysis steps were accomplished at laboratory temperature. The detector wavelength was fixed at $284 \mathrm{~nm}$.

\section{Synthesis of MIS Polymer}

The desired MIS sorbent has been synthesized as described in our previous work [8]. In brief, $0.15 \mathrm{~g}$ of isatin and $1000 \mu \mathrm{l}$ of concentrated $\mathrm{HCl}$ dissolved in deionized water. Isatin dummy template is structurally analogous to the HMF and possesses some advantages [9]: (1) Any leakage of the dummy template will not interfere with the analysis of the objective spices, (2) Its price is lower than the target molecule, and (3) It has usually less reactivity with the polymerization reagents. After $1 \mathrm{~h}, 30 \mathrm{ml}$ TEOS was added to the mixture, and the achieved precursor was heated in an oil bath at $70{ }^{\circ} \mathrm{C}$ for $48 \mathrm{~h}$. The obtained MIS sorbent was then grounded and sieved. Finally, the template molecules were completely extracted from the polymeric network by repeated soxhlet extraction utilizing water and different organic solvents. In addition, a non-imprinted sol-gel polymer (NIP) was synthesized utilizing the same method, in the absence of the dummy template.

\section{Online-SPE-HPLC Procedure}

The SPE minicolumn has been packed with $1.00 \mathrm{~g}$ of the synthesized MIS (or NIS). Then the minicolumn was placed instead of the sample loop of the HPLC system. in the load position, $2 \mathrm{ml}$ of sample solution was injected into the minicolumn at a flow rate of $2.0 \mathrm{ml}$ min-1 In this step, HMF was adsorbed on the MIS surface, and unwanted solutions were moved to the waste reservoir. In the inject position, the adsorbed HMF was eluted by the HPLC mobile phase and sent into the chromatographic separation column. After each cycle, the minicolumn has been thoroughly cleaned with methanol and deionized water to remove all unwanted contaminations and the solvent residue.

\section{Real Samples Analysis}

In this study, apple and cherry juices have been chosen as the real samples. The blank juices were prepared with fresh fruits and stored at $4{ }^{\circ} \mathrm{C}$ until the main analysis. The presence or absence of HMF in the samples was checked by the AOAC method [10]. All the juices were centrifuged for $10 \mathrm{~min}$ at 10,000 rpm and then filtered with $0.65-\mu \mathrm{m}$ Nylon membrane filters. Next, the clear supernatants were acidified with $\mathrm{HCl}$ solution to $\mathrm{pH}=4.0$. In the following, the samples were spiked with appropriate amounts of the HMF standard solution to estimate the method recovery. The chromatographic studied has been done as described in the previous section.

\section{Results and Discussion}

\section{Experimental Optimization}

Designing an extraction and pre-concentration procedure usually requires the optimization of the experimental factors influencing the method performance. In the present study, the $\mathrm{pH}$ of sample solutions, loading flow rate, and elution flow rate as the main parameters affecting the efficiency of the method have been optimized. The optimum amount of these parameters were: $\mathrm{pH}=4$, loading flow rate $=2 \mathrm{~mL} \mathrm{~min}^{-1}$ and elution flow rate $=1 \mathrm{~mL} \mathrm{~min}{ }^{-1}$. Patulin is the main interference during the HMF determination and generally prevents a dependable quantitative determination. HMF and patulin display similar chromatographic characterizations due to their chemical structures. Therefore, finding an appropriate eluent was a huge problem in this work. The proper eluent must be strong enough to desorb HMF from the SPE minicolumn, and also should be a suitable mobile phase to separate the HMF from patulin and the other compounds in the analytical column. For this purpose, several mobile phases (eluents) were studied. The highest recovery and separation efficiency was obtained utilizing $\mathrm{H}_{2} \mathrm{O}$ :THF $(80: 20 \mathrm{v} / \mathrm{v})$. 


\section{Imprinting Effect}

A NIP-packed minicolumn was used to estimate the presence of imprinted sites on the synthesized polymer. Then, a standard HMF solution $\left(0.10 \mu \mathrm{g} \mathrm{ml}^{-1}, \mathrm{pH}=4\right)$ was loaded onto the SPE column at the flow rate of $2.0 \mathrm{~mL} \mathrm{~min}^{-1}$ and continued by the proposed procedure. Comparison of the recoveries shows that the selectivity of imprinted polymer for HMF was significantly greater than nonimprinted one (Figure 1).

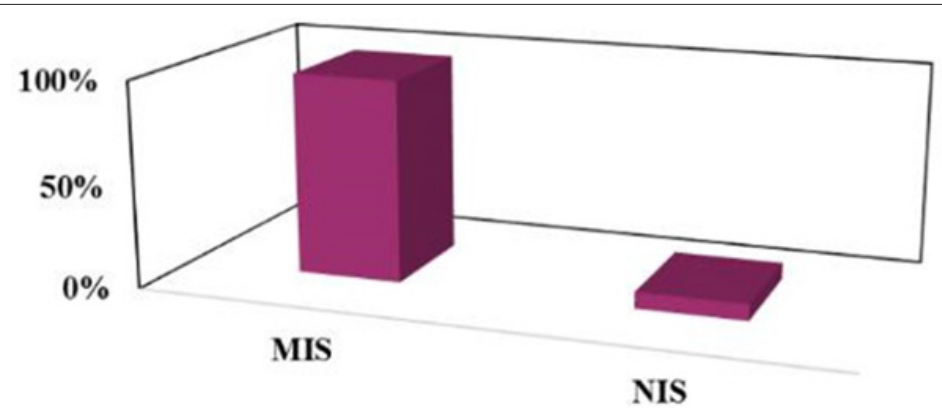

Figure 1: The imprinting effect of MIS versus NIS packed using the presented method.

\section{Analytical Figures of Merit}

The analytical figures of merit for the proposed online-SPEHPLC technique were investigated under the optimized conditions. The obtained calibration curves display excellent linearity for HMF over a concentration range of 0.03-0.45 $\mathrm{g} \mathrm{m} \mathrm{ml}^{-1}$ with a regression coefficient (R2) of more than 0.99 . The limit of detection (LOD) and limit of quantification (LOQ) for five repeated runs were 0.023 and $0.081 \mu \mathrm{g} \mathrm{ml}^{-1}$, respectively. The relative standard deviations (RSDs) of intraday and inter-day precision $(n=4)$ were $2.83 \%$ and $5.17 \%$, respectively.

\section{Commercial Samples Analysis}

The proposed approach was employed for the determination of HMF in some commercial apple and cherry juice samples. The juices were spiked with 0.05 and $0.20 \mathrm{\mu g} \mathrm{ml}^{-1}$ of HMF and analyzed by the developed online-SPE-HPLC procedures. Acceptable recoveries in all cases proved the high-ability of the developed method for the routine analysis of HMF in different standard/real samples (Table 1).

Table 1: Recovery results of HMF analysis from the spiked apple and cherry juices utilizing the proposed online-SPE-HPLC technique.

\begin{tabular}{|c|c|c|c|}
\hline Sample & Added $\left(\mu \mathbf{g ~ m l}^{-1}\right)$ & Found $\left(\mu \mathbf{~ m l}^{-1}\right)$ & Recovery (\%) \\
\hline Apple juice & 0 & 0.405 & - \\
\hline & 0.05 & 0.401 & 97.77 \\
\hline Cherry juice & 0.2 & 0.594 & 97.28 \\
\hline & 0 & - & - \\
\hline & 0.05 & 0.51 & 98.03 \\
\hline
\end{tabular}

\section{Conclusion}

Due to the importance of the SPE technique in the sampling and sample preparation and also the need to produce new SPE sorbents with better characteristics than the available ones, a new MIS sorbent was synthesized utilizing Isatin as a dummy template. The prepared polymer was used as the sorbent of the SPE-minicolumn in an online-SPE-HPLC technique. Rigidity, cost-effectiveness, high selectivity, good extraction capability, and high precision are the main properties of the proposed method. The method efficiency was investigated by the determination of HMF in some standard/ real samples. The experimental results were presented that the offered sorbent possesses strong application potentials in the highefficient SPE process.

\section{Acknowledgment}

The author gratefully acknowledges the Research Council of Islamic Azad University of Karaj for financial support.

\section{Competing Interests}

The author has declared no conflict of interest. 


\section{References}

1. Yalda Pasandideh, Habib Razmi (2021) Preparation of a new coating of graphene oxide/nickel complex on a nickelized metal surface for direct immersion solid phase microextraction of some polycyclic aromatic hydrocarbons. BMC Chemistry 15: 56.

2. Wen, Y (2020) Handbook of Nanomaterials in Analytical Chemistry: Recent advances in solid-phase extraction techniques with nanomaterials. Elsevier Science \& Technolog 57-73.

3. Yalda Pasandideh, Habib Razmi (2020) Introduction of a biowaste/ graphene oxide nanocomposite as a coating for a metal alloy based SPME fiber: Application to screening of polycyclic aromatic hydrocarbons. Arab J Chem 13: 8499-8512

4. Auréa Andrade-Eiroa, Moisés Canle, Valérie Leroy-Cancellieri, Víctor Cerdà (2016) Solid phase extraction of organic compounds: a critical review. Part ii. TrAC Trends Anal 80: 655-667.

5. Razmi H, Pasandideh Y (2020) Introduction of commercial heating elements of resistance metal alloys as the novel solid-phase microextraction fibers for chromatographic monitoring of organic pollutants. J Iran Chem Soc 17: 1111-1121

6. Mohammad Mahdi Moein, Abbi Abdel-Rehim, Mohamed AbdelRehim (2019) Recent Applications of Molecularly Imprinted Sol-Gel Methodology in Sample Preparation. Molecules 24: 2889.

7. Li, Jun; Xu, Youjie, Zhang, Meng, Wang, Donghai (2017) Determination of Furfural and 5-Hydroxymethylfurfural in Biomass Hydrolysate by HighPerformance Liquid Chromatography. Energy Fuels 31: 13769-13774.

8. Khorrami AR, Pasandideh Y (2016) Preparation of a novel sol-gel molecularly imprinted polymer with dummy template for online solidphase extraction of patulin from apple juice samples. Int J Anal Tech 2: $1-7$.

9. Yuanchen Liu, Dan Wang, Fuyou Du, Wenqianzheng, Zhiminliu, et al. (2019) Dummy-template molecularly imprinted micro-solid-phase extraction coupled with high-performance liquid chromatography for bisphenol A determination in environmental water samples. Microchem J145: 337-344.

10. 3rd AOAC Europe - EURACHEM Symposium 3 March (2005) Brussels. 